\title{
Plântulas de Syzygium malaccense (L.) Merr. \& L.M. Perry em função da coleta e
} temperaturas

\section{Seedlings of Syzygium malaccense (L.) Merr. \& L.M. Perry according to temperatures and temperatures}

\author{
Thaíse dos Santos Berto ${ }^{(1)}$, Luan Danilo Ferreira de Andrade Melo ${ }^{(12}{ }^{2}$, João Luciano de Andrade Melo \\ Junior ${ }^{\left(D^{2}\right.}$, Natália Marinho Silva Crisostomo ${ }^{(1}$, João Correia de Araújo Neto ${ }^{(2}{ }^{2}$, Vilma Marques \\ Ferreira (1) 2
}

${ }^{1}$ Alunas do Curso de Agroecologia, Universidade Federal de Alagoas, Campus de Engenharias e Ciências Agrárias, BR-104, Rio Largo, Alagoas. E-mails: thaiseberto7@gmail.com, natymarinhos@gmail.com; ${ }^{2}$ Professores da Universidade Federal de Alagoas, Campus de Engenharias e Ciências Agrárias, BR-104, Rio Largo, Alagoas. E-mails: luan.danilo@yahoo.com.br; luciiano.andrade@yahoo.com.br; jcanetto2@ hotmail.com; vmarques_ferreira@hotmail.com

\section{A R T I G O \\ Recebido: 08/05/2020 \\ Aprovado: 26/09/2020}

Palavras-chave

Fruticultura

Jambo vermelho

Myrtaceae

Produção vegetal

\section{Keywords}

Fruit growing

Jambo Vermelho

Myrtaceae

Vegetables production

\begin{abstract}
R E S U M O
O mercado do jambo (Syzygium malaccense (L.) Meer. \& L.M.Perry) tem crescido nos últimos anos, despertando a atenção de produtores brasileiros, que buscam a diversificação na produção e melhores preços no comércio. Hoje não há registro de nenhuma variedade, sendo a maioria dos pomares formados por mudas produzidas por sementes. Dessa forma, o trabalho teve como objetivo verificar a emergência de plântulas de jambeiro vermelho provenientes de sementes coletados no solo e na copa sob diferentes temperaturas. Foi adotado o delineamento experimental inteiramente casualizado, em esquema fatorial $2 \times 5$, sendo duas formas de coleta (solo e copa do jambeiro) e cinco temperaturas $\left(20,25,30,35,20-30{ }^{\circ} \mathrm{C}\right.$ ). Os parâmetros avaliados foram: teor de água, primeira contagem de emergência, emergência, índice de velocidade de emergência, comprimento da raiz e parte aérea e massa seca de plântulas. As sementes de jambo oriundas de frutos coletados no solo exibiram maior potencial fisiológico.
\end{abstract}

\begin{abstract}
A B S T R A C T
The market for jambo (Syzygium malaccense (L.) Meer. \& L.M. Perry) has grown in recent years, attracting the attention of Brazilian producers, who seek diversification in production and better prices in the fruit trade. Today there is no record of any variety, with most orchards formed by seedlings produced by seeds. Thus, the work aimed to verify the emergence of seedlings of jambo from seeds collected in the soil and in the crown under different temperatures. The work was conducted at the Plant Propagation Laboratory belonging to the Engineering and Agricultural Sciences Campus of the Federal University of Alagoas. A completely randomized experimental design was adopted, in a $2 \times 5$ factorial scheme, with two forms of collection (soil and canopy) and five temperatures $\left(20,25,30,35,20-30^{\circ} \mathrm{C}\right)$. The evaluated parameters were: water content, first emergency count, emergency, emergency speed index, root and shoot length and seedling dry mass. Jambo seeds from fruits collected in the soil exhibited greater physiological potential.
\end{abstract}

\section{INTRODUÇÃO}

O gênero Syzygium, ao qual pertence o jambeiro, está inserido na família Myrtaceae, sendo o maior gênero da família, com 1200 espécies (JUDD et al., 2009). Incluso no gênero, destaca-se a espécie jambeiro vermelho (Syzygium malaccense (L.) Meer. \& L.M.Perry) que têm como centro de origem o Continente Asiático, encontra-se em toda faixa tropical e, no Brasil, nas regiões da Amazônia, Cerrado, Pantanal e Mata Atlântica, com ampla comercialização nas regiões Norte e Nordeste (AUGUSTA et al., 2010).

O mercado do jambo tem crescido nos últimos anos, despertando a atenção de produtores brasileiros, que buscam a diversificação na produção e melhores preços no comércio dos frutos. O jambeiro vermelho apresenta excelente potencial de mercado, contudo são necessários mais estudos e pesquisas que viabilizem sua exploração comercial (NACATA, 2017).

Atualmente no Brasil não há registro de variedades da espécie, sendo a maioria dos pomares formados por mudas produzidas por sementes. Portanto, para que seja plausível explorar todo o seu potencial agrícola, o entendimento das relações que influenciam diretamente sua produção são essenciais para o estabelecimento da cultura (NACATA, 2017).

Trabalhos que retratam o potencial fisiológico de sementes auxiliam na determinação de padrões de plantas em

\section{Revista Verde}

ISSN 1981-8203

Pombal, Paraíba, Brasil v. 15, n.4, out.-dez., p.368-372, 2020 doi: $10.18378 /$ rvads.v15i4.7946

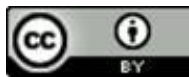


programas de melhoramento genético, além de fornecer subsídios para o manuseio e acondicionamento das sementes, padronizações de testes em laboratórios e melhoria na produção de mudas (MELO et al., 2018).

$\mathrm{O}$ processo de germinação e consequentemente emergência envolvem uma série de atividades metabólicas, onde ocorre uma sequência de reações químicas que apresentam exigências próprias quanto à temperatura, pois dependem das atividades enzimáticas específicas (MARCOS FILHO, 2015). O processo de germinação pode ser afetado por uma série de condições intrínsecas e extrínsecas, dentre as quais umidade, temperatura, substrato, luz e oxigênio. Entretanto, o conjunto é essencial para que o processo se realize normalmente, e a ausência de uma delas impeça a germinação da semente (CARVALHO; NAKAGAWA, 2012).

A forma como uma espécie responde a esses fatores define o sucesso no estabelecimento de suas plântulas, a descoberta da forma ideal de coleta de um fruto também pode auxiliar na identificação do ponto de maturidade fisiológica da semente, ou seja, quando ela atinge o máximo de germinação e vigor. Dentre as condições que afetam o processo germinativo, a temperatura é considerada um dos fatores que tem uma interferência significativa. As variações de temperatura influenciam a velocidade, a percentagem e a uniformidade de germinação (SILVA et al., 2016).

Dessa forma, o trabalho teve como objetivo verificar a emergência de plântulas de jambeiro vermelho provenientes de sementes coletados no solo e na copa sob diferentes temperaturas.

\section{MATERIAL E MÉTODOS}

O experimento foi conduzido no Laboratório de Propagação de Plantas, pertencente ao Campus de Engenharias e Ciências Agrárias (CECA)/ Universidade Federal de Alagoas (UFAL), Rio Largo, AL, Brasil. As sementes de jambo utilizadas foram extraídas de frutos maduros de jambeiros vermelhos, coletados de sete árvores localizadas nas dependências do CECA (solo e copa das árvores). Após a extração, as sementes foram lavadas em água corrente, secas em condição ambiente $\left( \pm 25^{\circ} \mathrm{C}\right)$ por 24 horas e desinfestadas, por imersão sequencial, durante 30 segundos, em álcool (70\%) e hipoclorito de sódio (2\%), a seguir sendo lavadas por 5 minutos em água corrente (MELO, 2017).

Após a desinfestação, as sementes foram homogeneizadas para formar dois lotes distintos e colocadas em bandejas plásticas, com substrato areia lavada (autoclavada), umedecida com água destilada a 60\% da capacidade de retenção de água, conforme Brasil (2009), em uma profundidade de $2 \mathrm{~cm}$ e submetidas a diferentes temperaturas: $20,25,30,35^{\circ} \mathrm{C}$ com 12 horas de luz e alternada $20-30^{\circ} \mathrm{C}\left(8\right.$ horas à $30^{\circ} \mathrm{C}$ e 16 horas à $\left.20^{\circ} \mathrm{C}\right)$, em câmaras de germinação tipo BOD (Biochemical Oxygen Demand). Para cada temperatura foram realizadas 4 repetições contendo 25 sementes. $\mathrm{O}$ experimento teve duração de aproximadamente 100 dias, sendo este período determinado pela estabilização completa da germinação.

Para a determinação do teor de água das sementes, foi utilizado o método de estufa a $105 \pm 3{ }^{\circ} \mathrm{C}$ por 24 horas, conforme prescrições das Regras para Análise de Sementes (BRASIL, 2009). Essa determinação foi realizada, por ocasião da instalação do ensaio, utilizando-se quatro amostras por tratamento.
Avaliou-se porcentagem diária de emergência, computando-se a primeira contagem de emergência (36 dias) e a emergência final (plântulas normais).

$O$ índice de velocidade de emergência (IVE) foi calculado com os dados diários de emergência obtidos, utilizando-se a fórmula de cálculo de Maguire (1962): IVE = $(\mathrm{E} 1 / \mathrm{N} 1)+$ $(\mathrm{E} 2 / \mathrm{N} 2)+(\mathrm{E} 3 / \mathrm{N} 3)+\ldots+(\mathrm{En} / \mathrm{Nn})$, sendo: E1, E2, E3, En = número de plântulas computadas na primeira, segunda, terceira e última contagem; N1, N2, N3, Nn = número de dias da semeadura à primeira, segunda e última contagem.

Ao final do teste de emergência, o hipocótilo e a raiz primária das plântulas normais de cada subamostras foram medidas com auxílio de régua graduada e os resultados expressos em centímetro por plântulas (MELO et al., 2017).

Logo após, as plântulas normais de cada repetição foram acondicionadas em sacos de papel, em seguida colocadas em estufa de ventilação forçada a $80{ }^{\circ} \mathrm{C}$, por um período de 24 horas. Transcorrido esse tempo, as amostras foram colocadas em dessecadores com sílica gel ativada e, pesadas em balança analítica com precisão de $0,0001 \mathrm{~g}$, e o resultado expresso em g/plântulas (CRISOSTOMO et al., 2018).

Foi adotado o delineamento experimental inteiramente casualizado (DIC), em esquema fatorial $2 \times 5$, sendo duas formas de coleta (solo e copa do jambeiro) e cinco temperaturas $\left(20,25,30,35\right.$ e $\left.20-30{ }^{\circ} \mathrm{C}\right)$. Quando houve significância do teste $\mathrm{F}$, as médias foram comparadas pelo teste de Tukey $5 \%$ de probabilidade O programa utilizado para as análises estatísticas foi o software SISVAR 5.6 (FERREIRA, 2011).

\section{RESULTADOS E DISCUSSÃO}

As sementes de jambo oriundas dos frutos que foram coletados no chão continham o maior teor de água inicial, como mostra a Tabela 1. De acordo com Guollo et al. (2016) os frutos abertos e em contato com solo podem necessitar de um período mais elevado para a perda de umidade e podem se umedecer principalmente à noite, em função da condensação do orvalho, fato ocorrido na presente pesquisa. Os frutos fechados tiveram sementes com menores teores de água, provavelmente por estarem presos à árvore, contudo, distante do solo, ficavam mais expostos às correntes de ar para perderem mais água. $\mathrm{O}$ conhecimento do ponto de maturidade fisiológica é primordial para se definir o ponto ideal de colheita (CARVALHO; NAKAGAWA, 2012).

Tabela 1. Teor de água de sementes de Syzygium malaccense (L.) Meer. \& L.M.Perry em função do tipo de coleta

\begin{tabular}{lc}
\hline Tipo de coleta & Teor de água $(\%)$ \\
\hline Solo & 30,2 \\
Copa & 27,2 \\
\hline
\end{tabular}

Estudos mostram informações a respeito do melhor momento para a coleta de sementes, como afirmam Vieira (2011) que para se obter máxima germinação em sementes de Anacardium occidentale L., estas devem ser colhidas com teor de água entre $10,4-15,5 \%$.

Para os resultados referentes ao vigor, observados na primeira contagem de emergência (PCE) (Tabela 2), observase que a combinação sementes coletadas no solo com temperatura constante de $30{ }^{\circ} \mathrm{C}$ foi responsável pelo maior valor, diferindo estatisticamente das demais combinações. 
Verifica-se ainda que não se obteve PCE para as sementes submetidas as temperaturas de $20^{\circ} \mathrm{C}$ (Solo e Copa) e $25^{\circ} \mathrm{C}$ (Copa).

Tabela 2. Primeira contagem de emergência (\%) de plântulas oriundas de sementes de Syzygium malaccense (L.) Meer. \& L.M.Perry em função do tipo de coleta e submetidas a temperaturas.

\begin{tabular}{lccccc}
\hline \multirow{2}{*}{ COLETA } & \multicolumn{5}{c}{ TEMPERATURA $\left({ }^{\circ} \mathrm{C}\right)$} \\
\cline { 2 - 6 } Solo & 20 & 25 & 30 & 35 & $20-30$ \\
\cline { 2 - 6 } Copa & $0 \mathrm{aD}$ & $37 \mathrm{aC}$ & $96 \mathrm{aA}$ & $37 \mathrm{aC}$ & $87 \mathrm{aB}$ \\
\hline $\mathrm{CV}(\%)$ & $0 \mathrm{aC}$ & $0 \mathrm{bC}$ & $85 \mathrm{bA}$ & $37 \mathrm{aB}$ & $85 \mathrm{bA}$ \\
\hline
\end{tabular}

Médias seguidas de mesma letra maiúscula na linha e minúscula na coluna não diferem a 5\% de probabilidade pelo teste de Tukey.

$\mathrm{O}$ fato de as sementes oriundas de frutos do solo muitas vezes apresentarem um maior percentual germinativo pode estar ligado à sua maturidade fisiológica. As sementes provindas das copas das árvores na maioria das vezes não se encontram no ponto de maturidade fisiológica (CARVALHO; NAKAGAWA, 2012; GUOLLO et al., 2016). Resultados diferentes foram descobertos em investigações realizadas com Anacardium occidentale L., quando se observou que os frutos colhidos na copa, apresentaram sementes com maior percentual germinativo, comparadas aquelas retiradas de frutos que estavam no solo (VIEIRA, 2011).

A faixa de temperatura ótima, para maioria das espécies, situa-se entre 20 e $30{ }^{\circ} \mathrm{C}$ (MARCOS FILHO, 2015). Para Larcher (2000) esta faixa se amplia ainda até os $35^{\circ} \mathrm{C}$. Em sementes Eugenia uniflora L. a utilização da temperatura de 30 ${ }^{\circ} \mathrm{C}$ proporcionou o maior número de sementes germinadas na primeira contagem (MELO et al., 2016). Brancalion et al (2008) informaram que a temperatura $30^{\circ} \mathrm{C}$ é a mais favorável para a germinação e consequentemente emergência da maioria das espécies vegetais, havendo relação entre a temperatura ótima e o bioma de ocorrência da espécie. Desta forma, o teste de primeira contagem de emergência é de suma relevância, pois estima a velocidade de emergência, apontando que quanto maior a germinação das sementes na primeira contagem, maior será seu vigor (PAIVA, 2012).

$\mathrm{Na}$ Tabela 3, encontra-se a porcentagem de emergência das plântulas de jambo, verifica-se que as sementes oriundas de frutos coletados do solo na temperatura constante de $30^{\circ} \mathrm{C}$ e alternada $20-30{ }^{\circ} \mathrm{C}$ apresentaram maiores porcentagens de emergência, não diferindo estatisticamente entre si. Costa et al. (2006) examinado o comportamento germinativo de sementes de Syzygium malaccense (L.) Merryl et Perry provindas de frutos coletados na copa, também verificaram resultados semelhantes.

Tabela 3. Emergência (\%) de plântulas oriundas de sementes de Syzygium malaccense (L.) Meer. \& L.M.Perry em função do tipo de coleta e submetidas a temperaturas.

\begin{tabular}{lccccc}
\hline \multirow{2}{*}{ COLETA } & \multicolumn{5}{c}{ TEMPERATURA $\left({ }^{\circ} \mathrm{C}\right)$} \\
\cline { 2 - 6 } Solo & 20 & 25 & 30 & 35 & $20-30$ \\
\cline { 2 - 6 } Copa & $0 \mathrm{aC}$ & $70 \mathrm{aB}$ & $100 \mathrm{aA}$ & $70 \mathrm{aB}$ & $94 \mathrm{aA}$ \\
$\mathrm{CV}(\%)$ & $0 \mathrm{aD}$ & $42 \mathrm{bC}$ & $94 \mathrm{bA}$ & $70 \mathrm{aB}$ & $90 \mathrm{bA}$ \\
\hline
\end{tabular}

Médias seguidas de mesma letra maiúscula na linha e minúscula na coluna não diferem a $5 \%$ de probabilidade pelo teste de Tukey.
Esses dados comprovam a amplitude de temperaturas em que pode ocorrer a germinação das sementes dessa espécie, o que possibilita maior capacidade de estabelecimento das plântulas em campo, tornando-as capazes de suportar as condições adversas do ambiente. Segundo Melo (2017) a temperatura ideal de germinação, geralmente, varia dentro da faixa de temperatura encontrada no local e na época ideal para emergência e estabelecimento das plântulas.

Foi observado no trabalho que as sementes de jambo germinaram em diferentes temperaturas, o que permite sua colonização em maior diversidade de habitat, facilitando assim sua dispersão. A amplitude térmica para germinação de sementes de uma espécie pode indicar a distância de uma semente enterrada em relação à superfície do solo, já que ela tende a diminuir com o aumento da profundidade (CARVALHO; NAKAGAWA, 2012).

No que se refere ao índice de velocidade de emergência (IVE) (Tabela 4), observou-se o maior índice com o uso da temperatura de $30^{\circ} \mathrm{C}$ nas sementes coletadas no solo, diferindo estatisticamente das demais combinações. Silva et al. (2014) estudando os efeitos da temperatura na germinação de sementes de Sideroxylon obtusifolium (Roem. \& Schult.) T.D. Penn. afirmaram que o índice em questão é linearmente dependente da temperatura, sendo bom para avaliar a ocupação de uma espécie em um determinado ambiente, pois a germinação rápida é característica de espécies cuja estratégia é de se estabelecer no ambiente o mais rápido possível aproveitando condições ambientais favoráveis.

Tabela 4. Índice de Velocidade de Emergência de plântulas de Syzygium malaccense (L.) Meer. \& L.M.Perry em função do tipo de coleta e submetidas a temperaturas.

\begin{tabular}{llcccc}
\hline \multirow{2}{*}{ COLETA } & \multicolumn{5}{c}{ TEMPERATURA $\left({ }^{\circ} \mathrm{C}\right)$} \\
\cline { 2 - 6 } & 20 & 25 & 30 & 35 & $20-30$ \\
\cline { 2 - 6 } Solo & $0,00 \mathrm{aC}$ & $3,67 \mathrm{aB}$ & $5,49 \mathrm{aA}$ & $3,67 \mathrm{aB}$ & $5,12 \mathrm{aB}$ \\
Copa & $0,00 \mathrm{aC}$ & $0,10 \mathrm{bC}$ & $5,12 \mathrm{bA}$ & $3,67 \mathrm{aB}$ & $5,00 \mathrm{bA}$ \\
\hline
\end{tabular}

$\mathrm{CV}(\%) \quad 9,76$

Médias seguidas de mesma letra maiúscula na linha e minúscula na coluna não diferem a $5 \%$ de probabilidade pelo teste de Tukey.

Ainda que os maiores percentuais de emergência serem encontrados em sementes provindas de coletas no solo, de acordo com Nogueira e Medeiros (2007) este método pode ter algumas desvantagens, já que, as sementes encontradas no chão estão mais passíveis à contaminação por fungos e ao ataque de insetos e roedores.

Ao se utilizar sementes provindas do solo, alguns cuidados devem ser tomados para evitar as desvantagens do processo, tais como, limpar o terreno e estender uma lona ou colocar coletores na projeção da copa para facilitar a coleta e diminuir os danos às sementes e realizar a coleta dos frutos ou sementes logo após sua dispersão, a fim de diminuir o ataque de fungos, insetos e roedores (FIGLIOLIA; PIÑARODRIGUES, 1995).

Quanto ao desenvolvimento inicial das plântulas, avaliado pelo comprimento da raiz primária e da parte aérea (Tabelas 5 e 6), observou-se que as maiores médias foram alcançadas quando empregada à temperatura de $30{ }^{\circ} \mathrm{C}$ combinada com as sementes coletadas no solo, diferindo estatisticamente das demais combinações. Provavelmente na referida temperatura tenha ocorrido uma degradação mais eficiente das reservas presentes nas sementes, o que acabou 
favorecendo o desenvolvimento das radículas e da parte aérea, uma vez que nessa fase todo o desenvolvimento das plântulas se deve à composição química das sementes (CARVALHO; NAKAGAWA, 2012).

Tabela 5. Comprimento radicular de plântulas (cm) oriundas de sementes de Syzygium malaccense (L.) Meer. \& L.M.Perry em função do tipo de coleta e submetidas a temperaturas.

\begin{tabular}{lccccc}
\hline \multirow{2}{*}{ COLETA } & \multicolumn{5}{c}{ TEMPERATURA $\left({ }^{\circ} \mathrm{C}\right)$} \\
\cline { 2 - 6 } Solo & 20 & 25 & 30 & 35 & $20-30$ \\
\cline { 2 - 6 } Copa & $0,00 \mathrm{aD}$ & $2,90 \mathrm{aC}$ & $6,00 \mathrm{aA}$ & $2,70 \mathrm{aC}$ & $4,70 \mathrm{aB}$ \\
\hline CV $(\%)$ & $0,00 \mathrm{aC}$ & $2,80 \mathrm{bB}$ & $4,70 \mathrm{bA}$ & $2,65 \mathrm{aB}$ & $4,20 \mathrm{bB}$ \\
\hline
\end{tabular}

Médias seguidas de mesma letra maiúscula na linha e minúscula na coluna não diferem a 5\% de probabilidade pelo teste de Tukey.

Tabela 6. Comprimento da parte aérea de plântulas $(\mathrm{cm})$ oriundas de sementes de Syzygium malaccense (L.) Meer. \& L.M.Perry em função do tipo de coleta e submetidas a temperaturas.

\begin{tabular}{lccccc}
\hline \multirow{2}{*}{ COLETA } & \multicolumn{5}{c}{ TEMPERATURA $\left({ }^{\circ} \mathrm{C}\right)$} \\
\cline { 2 - 6 } Solo & 20 & 25 & 30 & 35 & $20-30$ \\
\cline { 2 - 6 } Copa & $0,00 \mathrm{aC}$ & $5,30 \mathrm{aC}$ & $9,50 \mathrm{aA}$ & $5,30 \mathrm{aC}$ & $9,00 \mathrm{aB}$ \\
\hline $\mathrm{CV}(\%)$ & $0,00 \mathrm{aD}$ & $4,10 \mathrm{bC}$ & $9,00 \mathrm{bA}$ & $5,30 \mathrm{aB}$ & $8,90 \mathrm{bA}$ \\
\hline
\end{tabular}

Médias seguidas de mesma letra maiúscula na linha e minúscula na coluna não diferem a 5\% de probabilidade pelo teste de Tukey.

Melo et al. (2017) constatam que, além de pesado, o substrato areia pode apresentar a desvantagem de drenar excessivamente a água, ficando a parte superior ressecada, prejudicando a germinação e emergência. $\mathrm{Na}$ presente pesquisa, não foi visto ressecamento superficial desse substrato durante a realização dos testes de emergência. Segundo Rosseto et al. (2009) a determinação do comprimento de plântula é importante, conjuntamente com o teste de emergência, pois podem ocorrer sementes que apresentam alta porcentagem de emergência e baixo comprimento médio de plântulas, assim como baixa porcentagem de emergência, mas com alto comprimento médio de plântulas.

Ao avaliar a massa seca das plântulas (Tabela 7), o maior valor foi obtido quando se utilizou a combinação de sementes coletadas no solo em junção com a temperatura de $30^{\circ} \mathrm{C}$. Oliveira et al. (2014) relatam que as avaliações da massa seca são de grande importância na análise do desenvolvimento das plantas, assegurando o desenvolvimento inicial das plântulas no campo.

Tabela 7. Massa seca de plântulas (g) oriundas de sementes de Syzygium malaccense (L.) Meer. \& L.M.Perry em função do tipo de coleta e submetidas a temperaturas.

\begin{tabular}{lccccc}
\hline \multirow{2}{*}{ COLETA } & \multicolumn{5}{c}{ TEMPERATURA $\left({ }^{\circ} \mathrm{C}\right)$} \\
\cline { 2 - 6 } & 20 & 25 & 30 & 35 & $20-30$ \\
\cline { 2 - 6 } Solo & $0,00 \mathrm{aD}$ & $0,17 \mathrm{aC}$ & $0,53 \mathrm{aA}$ & $0,17 \mathrm{aC}$ & $0,51 \mathrm{aB}$ \\
Copa & $0,00 \mathrm{aD}$ & $0,10 \mathrm{bC}$ & $0,20 \mathrm{bA}$ & $0,17 \mathrm{aB}$ & $0,16 \mathrm{aB}$ \\
\hline CV $(\%)$ & 8,75 & \multicolumn{5}{c}{} \\
\hline
\end{tabular}

Médias seguidas de mesma letra maiúscula na linha e minúscula na coluna não diferem a 5\% de probabilidade pelo teste de Tukey.

\section{CONCLUSÃO}

Sementes de jambo oriundas de frutos coletados no solo exibiram maior potencial fisiológico.

\section{REFERÊNCIAS}

AUGUSTA, I. M.; NASCIMENTO do O. K.; COUTO G. P. A. M.; BORGES V. S. Caracterização física e química da casca e polpa de jambo vermelho (Syzygium malaccensis, (L.) Merryl \& Perry). Ciência e Tecnologia de Alimentos, v. 30, n. 4, p. 928-932, 2010. 10.1590/S0101-20612010000400014.

BRANCALION, P. H. S, NOVEMBRE, A. D. L.; RODRIGUES, R. R.; CHAMMA, H. M. C. P. Efeito da luz e de diferentes temperaturas na germinação de sementes de Heliocarpus popayanensis L. Revista Árvore, v.32, n.2, p.225232, 2008. 10.1590/S0100-67622008000200005.

BRASIL. Regras para Análise de Sementes. Ministério da Agricultura e Reforma Agrária. SNDA/DNPV/CLAV, 2009. Brasília. 365 p.

CARVALHO, N. M.; NAKAGAWA, J. Sementes: ciência, tecnologia e produção. Jaboticabal: FUNEP, 2012. 588p.

COSTA, R. S.; OLIVEIRA, I. V. M.; MÔRO, F. M.; MARTINS, A. B. G. Aspectos morfológicos e influência do tamanho da semente na germinação do jambo-vermelho. Revista Brasileira de Fruticultura, v. 28, n. 1, p. 117-120, 2006. 10.1590/S0100-29452006000100032.

CRISOSTOMO, N. M. S.; COSTA, E. A.; SILVA, C. L.; BERTO, T. S.; RAMOS, M. G. C.; MELO JUNIOR, J. L. A.; MELO, L. D. F. A.; ARAUJO NETO, J. C. Qualidade fisiológica de sementes de milho crioulo proveniente de diferentes localidades. Revista Craibeiras de Agroecologia, v. 3, n. 1, p. 6555-6560, 2018.

FERREIRA, D. F. Sisvar: a computer statistical analysis system. Ciência e Agrotecnologia, v. 35, n. 6, p. 1039-1042, 2011. 10.1590/S1413-70542011000600001.

FIGLIOLIA, M.B.; PIÑA-RODRIGUES, F.C.M. Manejo de sementes de espécies arbóreas. Instituto Florestal. Série registros, v.1, n.14, p.1-59, 1995.

GUOLLO, K.; FELIPPI, M.; POSSENTI, J. C. Germinação de sementes de Aspidosperma parvifolium A. DC. em função de diferentes formas de coleta. Ciência Florestal, v. 26, n. 3, p. 979-984, 2016. 10.5902/1980509824226.

JUDD, W. S.; CAMPBELL C.; STEVENS P. F.; DONOGHUE, M. J. Plant Systematics: A Phylogenetic Approach. 3 Ed. Massachusetts: Sinauer Associates Inc, 2009. $625 \mathrm{p}$.

LARCHER, W. Ecofisiologia vegetal. São Carlos: Rima, 2000. 531p.

MAGUIRE, J. D. Speed of germination: aid in selection and evaluating for seedling emergence and vigour. Crop Science, v.2, n.1, p.176-177, 1962. 
MARCOS FILHO, J. Fisiologia de sementes de plantas cultivadas. Piracicaba: FEALQ, 2015. 495p.

MELO, L. D. F. A.; MELO JUNIOR, J. L. A.; ARAUJO NETO, J. C.; FERREIRA, V. M. Potencial fisiológico de sementes de pitangueira obtidas em diferentes localidades e submetidas a temperaturas. Educação Ambiental em Ação, v.15, p.1-8, 2016

MELO, L. D. F. A. Morfometria, potencial fisiológico de sementes e desenvolvimento pós-seminal de Mimosa bimucronata (DC) O. KTZE. Tese: UFAL - Programa de PósGraduação em Agronomia (Produção Vegetal), Rio Largo, AL, 113 p., 2017.

MELO, L. D. F. A.; MELO JUNIOR, J. L. A.; MAGALHAES, I. D.; MEDEIROS, A. S.; MAIA JÚNIOR, S. O. CORDEIRO JUNIOR, J. J. F.; SILVA, A. C. Temperature and substrate effects on the germination of Caesalpinia ferrea Mart. Ex Tul. African Journal of Agricultural Research, v. 12, n. 1, p. 33483354, 2017. 10.5897/AJAR2017.12748.

MELO, L. D. F. A.; MELO JUNIOR, J. L. A.; FERREIRA, V. M. ; ARAUJO NETO, J. C. ; NEVES, M. I. R. S. . Biometric characterization and seed germination of giant mimosa (Mimosa bimucronata (DC) O. Kuntze). Australian Journal of Crop Science, v. 12, n.1, p. 108-115, 2018. 10.21475/ajcs.18.12.01.pne773.

NACATA, G. Jambeiro: Propagação, aspectos morfológicos e caracterização qualitativa. Dissertação (Produção Vegetal). Faculdade de Ciências Agrárias e Veterinárias - Unesp, Câmpus de Jaboticabal. 112p. 2017.

NOGUEIRA, A. C.; MEDEIROS, A. C. S. Coleta de sementes florestais nativas. Circular Técnica. Embrapa, Colombo-PR. 2007. 11 p.

OLIVEIRA, A. K. M.; RIBEIRO, J. W. F.; PEREIRA, K. C. L.; SILVA, C. A. A. Germinação de sementes de paineira-docampo (Eriotheca gracilipes (K. Schum.) A. Robyns) em diferentes temperaturas. Científica, v.42, n.4, p.316-324, 2014.

PAIVA, L. G. Tecnologia de sementes de Schinus terebinthifolius Raddi. Dissertação (Mestrado em Produção Agrícola). Universidade Federal Rural de Pernambuco, Garanhuns, PE, 68p. 2012.

RAMOS, A.; BIANCHETTI, A.; MARTINS, E G.; FOWLER, J. A. P.; ALVES, V. F. Substratos e temperaturas para a germinação de sementes de peroba (Aspidosperma polyneuron). Embrapa Florestas - Comunicado Técnico. Colombo: EMBRAPA-CNPF, 1995.

ROSSETO, J.; ALBUQUERQUE, M. C. F.; RONDON, N. R. M.; SILVA, I. C. O. Germinação de sementes de Parkia pendula (Willd.) Benth. ex Walp. (fabaceae) em diferentes temperaturas. Revista Árvore, v.33, n.1, p.47-55, 2009. 10.1590/S0100-67622009000100006.

SILVA, K. B.; ALVES, E. U.; OLIVEIRA, A. N. P.; SOUSA, N. A.; AGUIAR, V. A. Influência da luz e temperatura na germinação de sementes de quixaba. Revista AGROTEC, v. 35, n. 1, p 13-22, 2014. 10.25066/agrotec.v35i1.10254.

SILVA, D. L.; LUZ, G. R.; VELOSO, M. D. M.; FERNANDES, G. W.; NUNES, Y. R. F. Emergência e estabelecimento de plântulas de Guazuma ulmifolia LAM. Em função de diferentes tratamentos pré-germinativos. Ciência Florestal, v. 26, n. 3, p. 763-772, 2016. $\underline{10.5902 / 1980509824205}$

VIEIRA, F. E. R. Qualidade fisiológica de sementes de cajueiro, clone ccp-76, em função da forma de colheita e do tempo de armazenamento. Dissertação: Universidade Federal Rural do Semi-Árido - Mestrado em Fitotecnia (Área de concentração em Tecnologia de sementes), Mossoró, RN, 75 p., 2011. 